\title{
Endogenous Estradiol is Associated with Verbal Memory in Nondemented Older Men
}

\author{
Molly E. Zimmerman, Ph.D1, Richard B. Lipton, M.D ${ }^{1}$, Nanette Santoro, M.D2, Daniel S. \\ McConnell, Ph.D ${ }^{3}$, Carol A. Derby, Ph.D ${ }^{1,4}$, Mindy J. Katz, M.P. ${ }^{1}$, Khosrow Baigi, M.D ${ }^{1}$, and \\ Rachel Saunders-Pullman, M.D, M.P.H ${ }^{1,5}$ \\ ${ }^{1}$ Albert Einstein College of Medicine, Department of Neurology, Bronx, NY \\ 2 University of Colorado at Denver School of Medicine, Department of Obstetrics and \\ Gynecology, Denver, CO \\ ${ }^{3}$ University of Michigan School of Public Health, Department of Epidemiology, Ann Arbor, MI \\ ${ }^{4}$ Albert Einstein College of Medicine, Department of Epidemiology and Population Health, Bronx, \\ NY \\ ${ }^{5}$ Beth Israel Medical Center, Department of Neurology, New York, NY
}

\begin{abstract}
This study examined the relationship between endogenous hormones and cognitive function in nondemented, ethnically-diverse community-dwelling older men enrolled in the Einstein Aging Study (EAS). All eligible participants $(185$ men, mean age $=81$ years) received neuropsychological assessment (Free and Cued Selective Reminding Test (FCSRT), Logical Memory (LM), Trail Making Test B (TMTB), Block Design (BD)) and provided blood samples for hormonal assays (total estradiol, total testosterone, calculated free testosterone index). Linear regression analysis adjusted for age, education, body mass index, and cardiovascular comorbidities indicated that men with high levels of total estradiol demonstrated better FCSRT verbal memory performance ( $\beta=0.17, \mathrm{p}<0.02)$ compared to men with lower levels of total estradiol. The results remained unchanged when the model was further adjusted for ethnicity. We did not detect an association between testosterone and cognitive performance. These findings indicate that high levels of total estradiol in older men are associated with better performance on a cue-based, controlled learning test of verbal memory that is a sensitive predictor of dementia.
\end{abstract}

\section{Keywords}

Hormones; Cognition; Memory; Men; Older Adults; Aging; Testosterone; Estradiol

\section{INTRODUCTION}

The aging process is associated with heterogeneous declines in cognition both within and across individuals with normal cognitive abilities, mild cognitive impairment, and

Corresponding Author: Molly E. Zimmerman, Ph.D., Albert Einstein College of Medicine, Department of Neurology, 1165 Morris Park Avenue, Room 338, Bronx, NY 10461 USA, molly.zimmerman @einstein.yu.edu, tel: 718-430-3919, fax:718-430-3870.

Publisher's Disclaimer: This is a PDF file of an unedited manuscript that has been accepted for publication. As a service to our customers we are providing this early version of the manuscript. The manuscript will undergo copyediting, typesetting, and review of the resulting proof before it is published in its final citable form. Please note that during the production process errors may be discovered which could affect the content, and all legal disclaimers that apply to the journal pertain. 
dementia(Christensen, 2001; Jak et al., 2009; Zimmerman et al., 2006). Cognitive impairment in elderly adults is a critical public health concern that has been associated with functional decline, increased length of hospital stays, late life depression, and decreased quality of life(Alexopoulos et al., 2002; Gallo \& Lebowitz, 1999; Hoogerduijn, Schuurmans, Duijnstee, de Rooij, \& Grypdonck, 2007). Identifying predictors of cognitive change is a vital step toward improving the lives of the growing population of elderly individuals. Although the underlying biological causes of individual differences in cognitive function and decline are likely multifactorial, endogenous hormones may be an important determinant of cognitive performance in older adults (e.g., see(Yaffe et al., 2007)). A wide range of hormones exhibit age-associated alterations(Lamberts, van den Beld, \& van der Lely, 1997). Among elderly men, levels of the sex hormones testosterone and estradiol have been shown to decrease with age, while sex hormone-binding globulin (SHBG) levels increase with age(Moffat et al., 2002; Wolf \& Kirschbaum, 2002; Yaffe, Lui, Zmuda, \& Cauley, 2002).

Studies that examine relationships between cognitive function and endogenous testosterone levels among healthy nondemented older men have generally reported that higher levels of testosterone confer a cognitive advantage. An early study by Barrett-Connor and associates(Barrett-Connor, Goodman-Gruen, \& Patay, 1999) found that higher bioavailable testosterone was associated with better performance on tests of general mental status and episodic memory. Five-year intervals separated the time between acquisition of the blood samples and testing of cognitive function. Subsequent cross-sectional reports indicated that higher levels of testosterone in older men were related to better performance on tests of general mental status, executive function, and psychomotor speed(Yaffe et al., 2002), memory(Muller, Aleman, Grobbee, de Haan, \& van der Schouw, 2005; Thilers, Macdonald, \& Herlitz, 2006), processing speed(Martin, Wittert, Burns, Haren, \& Sugarman, 2007; Muller et al., 2005), and visuospatial abilities(Thilers et al., 2006). A recent study of 54 healthy older men reported a curvilinear relationship between free and bioavailable testosterone and performance on tests of working memory(Matousek \& Sherwin, 2010). In a longitudinal study, Moffat and colleagues(Moffat et al., 2002) reported that men with higher free testosterone levels exhibited better performance on tests of memory and visuospatial skills, and a reduced rate of decline on tests of visual memory over an average follow-up period of 10 years. In addition to these observational studies, Cherrier and colleagues conducted a series of exogenous testosterone supplementation randomized controlled trials in elderly men in which performance on neuropsychological tests was a primary outcome of interest. In hypogonadal men(Cherrier, Craft, \& Matsumoto, 2003), testosterone supplementation resulted in an increase in total testosterone and estradiol as well as verbal memory performance. In healthy older men(Cherrier et al., 2005), improvements in verbal memory performance were demonstrated in men who received testosterone supplementation, but not testosterone supplementation plus an aromatase inhibitor, suggesting that verbal memory performance in men may depend, in part, on the conversion of testosterone to estradiol. In contrast to these findings, Fonda and colleagues(Fonda et al., 2005) found no association between testosterone levels and cognition in a large sample of men across the age range following adjustment for multiple comorbidities.

Findings from studies that have examined estradiol and cognitive function in nondemented older men have been somewhat mixed. Higher total and bioavailable estradiol have been shown to be associated with poorer performance on a test of general mental status(BarrettConnor et al., 1999). Similarly, Geerlings and colleagues(Geerlings et al., 2006) found that higher bioavailable estradiol was associated with increased risk for cognitive decline and Alzheimer's Disease in older men in the Honolulu-Asia Aging Study using a general assessment of cognitive function administered three times over an average of six years. Others have also found a relationship between higher total and bioavailable estradiol and 
cognitive decline in older men that persisted after controlling for multiple covariates such as age and vascular risk factors(Muller, van den Beld, Grobbee, de Jong, \& Lamberts, 2009). In a sample of community-dwelling older men(Yaffe et al., 2002), higher total estradiol was associated with poorer performance on tests of visuomotor speed and executive function, but bioavailable estradiol was not associated with a test of general mental status. In contrast, a more recent longitudinal study by Yaffe and colleagues(Yaffe et al., 2007) identified a trend whereby older men with higher levels of bioavailable estradiol were less likely to experience cognitive decline on tests of general mental status and verbal memory. Still others have reported no relationship between cognitive performance and estradiol in older men (Fonda, Bertrand, O'Donnell, Longcope, \& McKinlay, 2005; Martin et al., 2007; Matousek \& Sherwin, 2010; Muller et al., 2005; Wolf \& Kirschbaum, 2002; Yaffe et al., 2002).

Such diversity of results among studies is likely due to several factors, including inclusion of individuals with varied age ranges as well as differences in hormonal assays, tests used for cognitive assessment, and sources of the study population (e.g., clinic- or communitybased). The objective of the current study was to augment our understanding of these complex relationships through an examination of endogenous hormones and cognitive function in nondemented older men. Our study represents a unique contribution to the literature in that our sample is relatively advanced in age (mean age $=81$ years, age range 70 to 94 years) and comprises volunteers from a systematically sampled, ethnically-diverse community who were carefully screened for dementia with comprehensive neuropsychological testing and neurological examination. We focused solely on men because relationships between hormones and cognition may differ as a function of sex. It was hypothesized that older men with high levels of total testosterone would demonstrate better cognitive performance on tests of executive function, visuospatial ability, and memory.

\section{MATERIALS AND METHODS}

\subsection{Participants}

One-hundred eighty-five participants in the current study were drawn from the Einstein Aging Study (EAS), a longitudinal community-based volunteer sample of individuals over the age of 70 residing in the Bronx, New York. The Albert Einstein College of Medicine institutional review board approved the use of human subjects included in this study. EAS study design and methods are described in more detail elsewhere(Lipton et al., 2003). Participants were recruited beginning in 1993 using systematic sampling methods that utilized voter registration lists for Bronx County. All participants completed a comprehensive neuropsychological evaluation of attention, executive functions, memory, language, and visuospatial construction abilities at annual visits. Depressive symptomatology was measured using the 15-item Geriatric Depression Scale (GDS(Sheikh $\&$ Yesavage, 1986)). All participants provided information regarding their past and current medical history that included self-report of myocardial infarction (MI), stroke, coronary artery bypass graft $(\mathrm{CABG})$ surgery, diabetes, hypertension, and smoking. Beginning in 2004, hormone assays were completed using blood samples collected at in-person clinic visits. Individuals who reported medical conditions that would interfere with completion of neuropsychological assessment, were non-English speakers, or who were institutionalized were excluded.

Global cognitive function was assessed with the Blessed Information-MemoryConcentration test (BIMC(Blessed, Tomlinson, \& Roth, 1968)). Cognitive status was determined by a study neurologist and neuropsychologist at a diagnostic case conference. Because the primary objective of this investigation was to examine the cross-sectional relationship between hormone levels and cognitive function in nondemented elderly men, 
we excluded persons with current dementia based on the criteria provided by the Diagnostic and Statistical Manual of Mental Disorders, $4^{\text {th }}$ Edition (DSM-IV)(American, 1994) Therefore, 185 nondemented elderly men with available memory and hormone data were included in the current study.

Mild cognitive impairment (MCI) is a clinical term that is commonly used to characterize a transition state between normal cognitive aging and dementia. Based on Petersen criteria(Petersen et al., 1999), three of the participants in this sample met diagnostic criteria for amnestic mild cognitive impairment (MCI) as evidenced by 1) objective memory impairment (free recall FCSRT score $\leq 24$; described in detail below) and 2) a subjective memory complaint. FCSRT free recall memory impairment cut-scores were derived from prior analyses(Grober \& Kawas, 1997; Grober, Lipton, Hall, \& Crystal, 2000).

\subsection{Neuropsychological Evaluation}

Verbal Memory-Consistent with the hypotheses of the current study, free recall from the FCSRT was selected from the EAS neuropsychological battery as a verbal memory variable of interest(Buschke, 1984; Grober \& Buschke, 1987). The FCSRT is a test that controls attention and strategy use in an encoding phase to maximize learning. In the first part of the task, participants name 16 objects that are pictorially displayed. They are then presented with the same 16 objects (four at a time in a $2 \times 2$ grid) and asked to identify each object following a cue that is a categorical prompt. In the free recall condition, a measure of selforganized retrieval, the participant is immediately asked to recall the 16 objects. If the participant fails to correctly recall an object, they are provided with a category cue to test cued recall. There are a total of three free and cued recall trials; scores range from zero to 48.

The Logical Memory (LM) subtest from the Wechsler Memory Scale - Revised(Wechsler, 1987) is a verbal memory test in which participants hear two contextually related short stories and are immediately asked to recall details of each story. Scores range from zero to 50 .

Executive Function-Trail Making Test part B (TMTB)(Battery, 1944) is a measure of executive function involving mental flexibility, set-shifting, and concept formation. Scores for TMTB are provided as seconds to task completion.

Visuospatial Construction-The Block Design (BD) subtest from the Wechsler Adult Intelligence Scale - Third Edition (WAIS-III(Wechsler, 1997)) was selected to examine the cognitive domain of visuospatial abilities. This test requires manipulation of red and white blocks in order to match a sample and it involves use of visuospatial and construction skills. Scores range from zero to 68 .

\subsection{Hormonal Assays}

All blood specimens were drawn in the morning. Assays were performed on stored serum samples collected between 2004 and 2007 and stored in separate aliquots at $-80^{\circ}$.

Testosterone and SHBG assays were performed in the laboratory of Dr. Santoro using DPC reagents (Los Angeles, CA) for testosterone and DELFIA (Perkin-Elmer, Gaithersburg, MD) for SHBG. Coefficients of variation were calculated from within and between assay quality control duplicates at 3 levels for SHBG and at 2 levels for testosterone. Respective intra- and interassay coefficients of variation were $4.3 \%$ and $8.6 \%$ for SHBG level, and $9.0 \%$ and $10.6 \%$ for total testosterone level. The sensitivity of the testosterone assay was $0.04 \mathrm{ng} / \mathrm{mL}$. For each specimen, a free testosterone index (FTI) was calculated using the modified Sodergard equation(Sodergard, Backstrom, Shanbhag, \& Carstensen, 1982): 
FTI=total testosterone/SHBG $\times 100$. Estradiol assays were performed in the CLASS laboratory at the University of Michigan. Serum estradiol concentrations were measured with a direct, modified off-line ACS:180 (E2-6) immunoassay (Bayer Diagnostics Corporation, Norwood, MA) that has been well-characterized(England, Parsons, Possley, McConnell, \& Midgley, 2002). Intra- and interassay coefficients of variation were $6.4 \%$ and $10.6 \%$, respectively, over the assay range of $20-500 \mathrm{pg} / \mathrm{ml}$. In secondary analyses, a free estradiol index (FEI) was calculated: FEI=100 x total estradiol/272.11 x SHBG(Thurston et al., 2009; van den Beld, de Jong, Grobbee, Pols, \& Lamberts, 2000).

\subsection{Statistical Analyses}

A dichotomous cardiovascular comorbidity score (CVD) was calculated to identify all participants with a past history of either myocardial infarction, stroke, or CABG. Age, education, and body mass index (BMI), were examined as potential covariates using Pearson product-moment correlation coefficients. Ethnicity was examined as a potential covariate using independent t-tests.

A series of separate linear regression analyses adjusting for age, education, BMI, and CVD were performed to examine relationships between endogenous hormone levels and cognitive performance in nondemented older men. The dependent variable was performance on a cognitive test (FCSRT, LM, TMTB, or BD) and the independent variable was a dichotomized hormone level (High Estradiol, Low Estradiol, High Testosterone, Low Testosterone, High FTI, or Low FTI). Because hormone measures were non-normally distributed, these data were classified by quartile (see(Barrett-Connor et al., 1999)) and then dichotomized using two different clinically relevant categorizations that maximized use of all available data. The High Hormone classification was defined as quartile 4 versus quartiles 1,2, and 3, while the Low Hormone classification was defined as quartile 1 versus quartiles 2, 3, and 4. Table 1 depicts endogenous hormone levels for the total sample and each quartile.

In secondary analyses, separate linear regression models adjusting for age, education, BMI, and CVD were performed in which the dependent variable was performance on the FCSRT and the independent variable was either SHBG or the calculated FEI. SHBG and FEI were dichotomized using identical methods described above. In addition, because previous studies(Martin et al., 2007; Muller et al., 2005) have found curvilinear associations between optimal levels of testosterone and cognitive function in older men, we repeated primary analyses with the same dependent variables (FCSRT, LM, TMTB, or BD) and covariates (age, education, BMI, and CVD) with a substitution of a quadratic term for testosterone as the independent variable.

\section{RESULTS}

\subsection{Sample Characteristics}

Endogenous hormone levels are presented in Table 1. One way ANOVA pairwise comparisons showed that all mean hormone level quartiles were significantly different from each other at the $\mathrm{p}<0.01$ level. There were a total of $36(20 \%)$ men who were defined as hypogonadal using the criteria of total testosterone $<3.2 \mathrm{ng} / \mathrm{mL}$ (Wu et al., 2010). Of the 94 men over the age of 80 years, 19 (20\%) met criteria for hypogonadism. Sample demographics, cognitive test performance, and medical history are presented by the total sample and total estradiol and total testosterone quartile in Table 2 and Table 3, respectively. Demographic characteristics (age, education, ethnicity) of men included in the analysis were consistent with those of older men from the EAS cohort recruited over the same time period who were excluded from the analysis because they did not provide blood samples for 
hormonal assays $(\mathrm{n}=43)$. Participants performed in the average range on neuropsychological tests(Holtzer, Goldin, \& Zimmerman, 2007;Steinberg, Bieliauskas, Smith, \& Ivnik, 2005). The sample comprised predominantly Caucasian males with an average 81 years of age (range: 70-94) and 14 years of education. No participant scored above the cut-off of 7/15 for significant depressive symptomatology on the GDS(Sheikh \& Yesavage, 1986). Participants reported prevalence rates of $16.8 \%$ myocardial infarction, $12 \%$ stroke, $17.4 \%$ CABG, $22.3 \%$ diabetes, 59.8\% hypertension, and 62.6\% smoking history. Sixty-five (35.3\%) participants endorsed a past history of either MI, stroke, or CABG (CVD index).

\subsection{Simple Correlations}

Pearson correlation coefficients among age, education, BMI, cognitive, and hormone variables are presented in Table 4 . As expected, younger participants demonstrated better performance on the FCSRT $(r=-0.25, \mathrm{p}<0.01), \mathrm{LM}(\mathrm{r}=-0.16, \mathrm{p}<0.04)$, and TMTB $(\mathrm{r}=0.30$, $\mathrm{p}<0.01)$. Participants with greater education also demonstrated better performance on LM $(\mathrm{r}=0.44, \mathrm{p}<0.01)$, TMTB $(\mathrm{r}=-0.41, \mathrm{p}<0.01)$, and $\mathrm{BD}(\mathrm{r}=0.33, \mathrm{p}<0.01)$, but there was no relationship between education and FCSRT performance. Caucasian participants performed better on LM $(t=4.78, p<0.01)$, TMTB $(t=-4.57, \mathrm{p}<0.01)$, and $\mathrm{BD}(\mathrm{t}=3.91, \mathrm{p}<0.01)$ compared to African-Americans and other ethnic groups. Partial correlations controlling for age and education did not change the strength of association between ethnicity and performance on the cognitive tests. BMI was not associated with any of the primary cognitive or hormone variables of interest. Based on these correlational relationships, age and education were used as covariates in all subsequent regression analyses. BMI and CVD were also included as covariates as they have been shown in prior studies to have independent effects on the primary variables of interest(Hogervorst, Matthews, \& Brayne, 2010). In order to minimize the number of terms in analysis models, the effect of ethnicity was only considered as an additional covariate in models that were significant following control of age, education, BMI, and CVD.

\subsection{Hormones and Verbal Memory}

A linear regression model adjusting for age, education, BMI, and CVD indicated that men with high levels of total estradiol demonstrated better FCSRT verbal memory performance $(\beta=0.17, \mathrm{p}<0.02)$ compared to men with lower estradiol. Age was also a significant correlate of FCSRT verbal memory performance $(\beta=-0.23, p<0.01)$. Figure 1 depicts FCSRT free recall means (adjusted for age, education, BMI, and CVD) and standard errors for high (quartile 4; $n=45$ ) versus low (quartiles 1, 2, and 3; $n=139$ ) estradiol groups. The high estradiol group demonstrated a mean FCSRT free recall performance of 29.55 (maximum score $=48$ ) and the low estradiol group had a score of 31.89. Compared to published FCSRT normative data from our larger community-based sample(Holtzer et al., 2007), the high estradiol group FCSRT mean score was well within the normal range and the low estradiol group FCSRT mean score was approximately one standard deviation below the normal mean. When the model was further adjusted for ethnicity; high estradiol remained a significant correlate of FCSRT verbal memory performance $(\beta=0.18, p<0.02)$. Neither testosterone, FTI, or the quadratic testosterone term were significant correlates of FCSRT performance. In addition, none of the models for the prediction of LM verbal memory performance by hormone level (estradiol, testosterone, FTI, or quadratic testosterone) controlling for age, education, BMI, and CVD were significant.

To further explore the primary finding of a relationship between high total estradiol and FCSRT performance, secondary analyses were conducted to better understand the contribution of SHBG to the total estradiol measurement. Separate linear regression models adjusting for age, education, BMI, and CVD indicated that neither SHBG level (high SHBG: 
$\beta=-0.03, p<0.68$; low SHBG: $\beta=0.08, p<0.33$ ) nor FEI level (high FEI: $\beta=0.10, p<0.21$; low FEI: $\beta=-0.05, p<0.50$ ) were associated with FCSRT verbal memory performance.

\subsection{Hormones and Executive Function and Visuospatial Construction}

None of the models of the association of TMTB or BD with hormone level (estradiol, testosterone, FTI, or quadratic testosterone) controlling for age, education, BMI, and CVD were significant.

\subsection{Memory Impairment}

As described previously, three participants met diagnostic criteria for amnestic MCI(Petersen et al., 1999). To further explore the extent to which memory impairment may be driving the findings, linear regressions were repeated with the removal of the MCI participants and results remained unchanged (data not shown).

\section{DISCUSSION}

This goal of this cross-sectional study was to better understand the contribution of endogenous sex hormones to memory, visuospatial construction, and executive function in nondemented community-dwelling older men. A unique aspect of this sample was that the study participants were relatively advanced in age with a mean of 81 years of age, ranging from 70 to 94 years of age. The findings indicate that high levels of total estradiol were associated with better performance on a test of verbal memory in older men even after controlling for the effects of age, education, body mass index, cardiovascular comorbidities, and ethnicity. The results remained unchanged when individuals with mild cognitive impairment were removed from the sample. There were no significant linear or curvilinear relationships between testosterone levels, a free testosterone index, or a free estradiol index and performance on cognitive tests.

Our finding of an association between estradiol and FCSRT verbal memory abilities in older men differs from previous studies that have reported relationships between higher estradiol and poorer performance on tests of general mental status(Barrett-Connor et al., 1999), visuomotor speed, and executive function(Yaffe et al., 2002), and greater risk of cognitive decline and Alzheimer's Disease(Geerlings et al., 2006; Muller et al., 2009). Several other studies found no relationship between estradiol and memory function in older men(BarrettConnor et al., 1999; Fonda et al., 2005; Martin et al., 2007; Matousek \& Sherwin, 2010; Muller et al., 2005; Wolf \& Kirschbaum, 2002; Yaffe et al., 2002). Discrepancies among findings may be explained, in part, by differences in the neuropsychological assessment of verbal memory. Previous reports have employed tests of verbal memory that involve the presentation of paired associates, short stories, or a list of words. These types of verbal memory tests are useful indicators of memory for novel information and are similar to the Logical Memory test utilized in the current study, which was notably not associated with sex hormone levels in older men. The FCSRT free recall measure, however, is unique in that it utilizes a controlled-learning paradigm that effectively focuses attention and provides cuebased strategies in order to maximize learning and recall of verbal information. FCSRT performance has been shown to have high diagnostic sensitivity and specificity for the identification of individuals with prodromal or early stage Alzheimer's Disease(Grober et al., 2000; Habert et al., 2009; Sarazin et al., 2007). Thus, our findings suggest that total estradiol levels in older men are associated with performance on a cued controlled-learning verbal memory task that is a sensitive indicator of the development of subtle early memory difficulties in individuals who later develop Alzheimer's Disease. 
Sex hormone-binding globulin (SHBG) is a binding protein that is a powerful proxy marker for insulin sensitivity and diabetes risk(Stellato, Feldman, Hamdy, Horton, \& McKinlay, 2000). Hepatic production of SHBG is coordinately regulated by both insulin and sex hormones. Elevated insulin is associated with lower SHBG and low insulin levels are related to higher circulating SHBG(Peiris et al., 1993). In addition, estradiol increases levels of SHBG while testosterone decreases levels of SHBG. The total estradiol measurement utilized in the current study comprised both SHBG and freely-bound estradiol. In order to explore the contribution of SHBG to our primary findings, we performed secondary analyses that examined relationships between FCSRT verbal memory performance and both SHBG and a calculated free estradiol index (FEI), which takes into account the proportion of estradiol that is protein bound and therefore believed to be unavailable for tissue uptake. The lack of association of SHBG or FEI with FCSRT performance is somewhat unexpected given that higher estradiol would be expected to upregulate SHBG. This finding suggests one of two possibilities. The first is that free estradiol alone does not affect memory performance in our sample of older men. The second and perhaps more likely possibility is that the coordinate regulation of SHBG by metabolic factors such as insulin sensitivity and overall hepatic function affect SHBG and FEI but not necessarily total circulating estradiol, and that these factors interact in a complex way that our methodology is unable to discern, despite controlling for BMI and CVD comorbidities. The specificity of our findings suggests that it is the dynamic combination of improved general health and higher production of estradiol in older men that may promote performance on a cued verbal memory task.

We found no evidence of an association between total testosterone or a calculated free testosterone index and performance on cognitive tests that assessed executive function, verbal memory, and visuospatial function. Our finding that estradiol, but not testosterone, was related to performance on a test of verbal memory (i.e., the FCSRT) that has been shown to be sensitive to prodromal Alzheimer's Disease suggests that testosterone may not be involved in the subtle early memory difficulties of elderly men. Similar to our results, Fonda and colleagues(Fonda et al., 2005) found no association between testosterone levels and cognition in a large sample of men across the age range following adjustment for multiple comorbidities. However, several other research groups found that higher levels of testosterone confer an advantage on performance on tests of general mental status, executive function, and psychomotor speed(Yaffe et al., 2002), memory(Muller et al., 2005; Thilers et al., 2006), processing speed(Martin et al., 2007; Muller et al., 2005), and visuospatial abilities(Thilers et al., 2006). One study reported a relationship between high testosterone levels and speed of information processing in healthy eldery men aged 61 to 72 years, but not in other age groups(Hogervorst, De Jager, Budge, \& Smith, 2004). Matousek and Sherwin(Matousek \& Sherwin, 2010) recently reported a curvilinear relationship in healthy older men where free and bioavailable testosterone were related to working memory performance. Conversely, other studies have reported that higher testosterone levels were associated with poorer performance on a test of verbal fluency(Wolf \& Kirschbaum, 2002) and tests of verbal memory and executive function(Martin et al., 2007). It is also possible that sex differences in cognitive function may have contributed to these discrepant findings. In has been reported that testosterone levels in men are associated with better performance on tests of visuospatial skills and poorer performance on tests of verbal memory (see review in(Janowsky, 2006)). It is also important to note that twenty percent of our sample met criteria for hypogonadism (total testosterone < $3.2 \mathrm{ng} / \mathrm{mL}$; (Wu et al., 2010)) which may have contributed to our inability to observe significant relationships between testosterone levels and cognition (see(Matousek \& Sherwin, 2010) for discussion). In addition, studies showing relationships between testosterone levels and cognitive function that vary as a function of age group(Hogervorst et al., 2004) suggest that the relatively advanced age of our participants may also have impacted our ability to observe associations. 
It is important to consider these findings within the context of interactive relationships between the production and regulation of different sex hormones. In men, circulating estradiol is derived from peripheral conversion of testosterone or adrenal androgen precursors, such as androstenedione and dehydroepiandrosterone (DHEA)(Lombardi et al., 2001). CYP19, the aromatase enzyme, is responsible for this bioconversion. In many cases, when testosterone is elevated, estradiol is also elevated, because of their precursor-product relationship. This appears to be the case in prostate cancer, wherein both elevated testosterone and elevated estradiol are related to disease risk(Carruba, 2007). One might therefore expect that if our findings were due solely to elevations in peripheral estradiol, a similar relationship would have been found with testosterone. However, there are some situations in which this is not the case. Peripheral estradiol appears important in preservation of bone mineral density in men, apart from testosterone(Vandenput \& Ohlsson, 2009), and indicates that the roles of both testosterone and estradiol need not be considered together. Recent findings that estrogen receptor-alpha mediates neuroprotection against ischemia in aging animals(Jover-Mengual, Zukin, \& Etgen, 2007), and that aromatase activity in reactive neuroglia contributes to neuronal repair(Azcoitia, Sierra, Veiga, \& Garcia-Segura, 2003) imply that estradiol, but not testosterone, may operate selectively in the brain.

Several biological studies support the mechanistic plausibility of our primary finding of an association between estradiol and verbal memory performance. Estrogen receptors are located in the hippocampus, a brain area that supports verbal memory abilities in older adults across the spectrum of cognitive status(Osterlund, Grandien, Keller, \& Hurd, 2000; Savaskan, Olivieri, Meier, Ravid, \& Muller-Spahn, 2001; Zimmerman et al., 2008). Studies have also shown that postmenopausal women with Alzheimer's disease exhibit lower levels of estradiol(Manly et al., 2000; Tsolaki et al., 2005). In addition, estrogen facilitates axonal sprouting and dendritic spine formation in rat CA1 hippocampal neurons(Matsumoto, 1991; McEwen, 1999), and estradiol treatment has been shown in in-vitro studies to protect neuronal cells from an accumulation of beta-amyloid, a protein thought to play an important role in the development of Alzheimer's disease(Xu et al., 1998).

Strengths of our study include a comprehensive assessment of sex hormone levels and cognitive function across several domains in a community-based study of ethnically-diverse nondemented older men. There are also several limitations to our study that warrant further discussion. We employed a cross-sectional design to examine our primary variables of interest; therefore, causality of the observed relationships cannot be inferred from our results. Additional longitudinal studies are needed to clarify the role of hormone levels on the development of cognitive impairment and dementia in older adults. Further, we chose to focus our study on men; therefore, our findings are generalizable only to nondemented men living independently in communities. While it is generally considered a strength that our sample comprised men of a relatively advanced age, it may also be a limitation for several reasons. First, because at least $20 \%$ of our participants were hypogonadal, there may be less variability in hormone measurements, thereby decreasing our power to detect a difference across eugonadal ranges(Hogervorst et al., 2004). In addition, because age itself is a risk factor for cognitive decline, it may be that the older men in our sample were already in a more vulnerable state as the biological stressors which precede and contribute to cognitive compromise may have already occurred. That is, if a healthy cell hypothesis(Brinton, 2005) holds whereby healthy cells respond beneficially to hormones but damaged cells respond negatively, we may be less likely to detect an effect because there is presumably a greater mix of healthy and damaged cells in an elderly population. Finally, as mentioned previously, sex hormones exert their biological influence within an interactive and dynamic framework that may not have been adequately reflected in our study design. Additional studies employing experimental designs and randomized controlled trials, such as the work by Cherrier and colleagues (for example, see(Cherrier et al., 2003; Cherrier et al., 2005; 
Cherrier et al., 2007)), are necessary to fully explore the dynamics of these steroids and their effect on the cognitive abilities of older men at varying age ranges.

In summary, we found that high levels of total estradiol were associated with better performance on a test of verbal memory in nondemented older men. Our results support the hypothesis that endogenous sex hormones may play a role in cognitive function in older men. In addition, our findings suggest that longitudinal studies assessing the relationship between estradiol levels and change in verbal memory in older men are warranted.

\section{Acknowledgments}

The work presented in this paper was supported by National Institutes of Health Grant AG03949 (RBL) and Grant NS047256 (RSP). The Authors would like to thank Charlotte Magnotta for assistance with participant recruitment; Betty Forro, Alicia Gomez, and Mary Joan Sebastian for assistance with neuropsychological assessment; and the study participants who generously gave their time in support of this research.

\section{References}

Alexopoulos GS, Buckwalter K, Olin J, Martinez R, Wainscott C, Krishnan KR. Comorbidity of late life depression: an opportunity for research on mechanisms and treatment. Biological Psychiatry. 2002; 52(6):543-558. [PubMed: 12361668]

American, PA. Diagnostic and statistical manual of mental disorders. 4. Washington, D.C: American Psychiatric Association; 1994.

Azcoitia I, Sierra A, Veiga S, Garcia-Segura LM. Aromatase expression by reactive astroglia is neuroprotective. Ann N Y Acad Sci. 2003; 1007:298-305. [PubMed: 14993062]

Barrett-Connor E, Goodman-Gruen D, Patay B. Endogenous sex hormones and cognitive function in older men. J Clin Endocrinol Metab. 1999; 84(10):3681-3685. [PubMed: 10523014]

Battery, AIT. Manual of Directions and Scoring. Washington, D.C: War Department, Adjutant Generals Office; 1944.

Blessed G, Tomlinson BE, Roth M. The association between quantitative measures of dementia and of senile change in the cerebral grey matter of elderly subjects. Br J Psychiatry. 1968; 114(512):797811. [PubMed: 5662937]

Brinton RD. Investigative models for determining hormone therapy-induced outcomes in brain: evidence in support of a healthy cell bias of estrogen action. Ann N Y Acad Sci. 2005; 1052:57-74. [PubMed: 16024751]

Buschke H. Cued recall in amnesia. J Clin Neuropsychol. 1984; 6(4):433-440. [PubMed: 6501581]

Carruba G. Estrogen and prostate cancer: an eclipsed truth in an androgen-dominated scenario. J Cell Biochem. 2007; 102(4):899-911. [PubMed: 17786930]

Cherrier MM, Craft S, Matsumoto AH. Cognitive changes associated with supplementation of testosterone or dihydrotestosterone in mildly hypogonadal men: a preliminary report. J Androl. 2003; 24(4):568-576. [PubMed: 12826696]

Cherrier MM, Matsumoto AM, Amory JK, Ahmed S, Bremner W, Peskind ER, et al. The role of aromatization in testosterone supplementation: effects on cognition in older men. Neurology. 2005; 64(2):290-296. [PubMed: 15668427]

Cherrier MM, Matsumoto AM, Amory JK, Johnson M, Craft S, Peskind ER, et al. Characterization of verbal and spatial memory changes from moderate to supraphysiological increases in serum testosterone in healthy older men. Psychoneuroendocrinology. 2007; 32(1):72-79. [PubMed: 17145137]

Christensen H. What cognitive changes can be expected with normal ageing? Aust N Z J Psychiatry. 2001; 35(6):768-775. [PubMed: 11990887]

England BG, Parsons GH, Possley RM, McConnell DS, Midgley AR. Ultrasensitive semiautomated chemiluminescent immunoassay for estradiol. Clin Chem. 2002; 48(9):1584-1586. [PubMed: 12194939] 
Fonda SJ, Bertrand R, O’Donnell A, Longcope C, McKinlay JB. Age, hormones, and cognitive functioning among middle-aged and elderly men: cross- sectional evidence from the Massachusetts Male Aging Study. J Gerontol A Biol Sci Med Sci. 2005; 60(3):385-390. [PubMed: 15860479]

Gallo JJ, Lebowitz BD. The epidemiology of common late-life mental disorders in the community: themes for the new century. Psychiatr Serv. 1999; 50(9):1158-1166. [PubMed: 10478901]

Geerlings MI, Strozyk D, Masaki K, Remaley AT, Petrovitch H, Ross GW, et al. Endogenous sex hormones, cognitive decline, and future dementia in old men. Ann Neurol. 2006; 60(3):346-355. [PubMed: 16865684]

Grober E, Buschke H. Genuine memory deficits in dementia. Developmental Neuropsychology. 1987; 3:13-36.

Grober E, Kawas C. Learning and retention in preclinical and early Alzheimer's disease. Psychology \& Aging. 1997; 12(1):183-188. [PubMed: 9100279]

Grober E, Lipton RB, Hall C, Crystal H. Memory impairment on free and cued selective reminding predicts dementia. Neurology. 2000; 54(4):827-832. [PubMed: 10690971]

Habert MO, Horn JF, Sarazin M, Lotterie JA, Puel M, Onen F, et al. Brain perfusion SPECT with an automated quantitative tool can identify prodromal Alzheimer's disease among patients with mild cognitive impairment. Neurobiol Aging. 2009

Hogervorst E, De Jager C, Budge M, Smith AD. Serum levels of estradiol and testosterone and performance in different cognitive domains in healthy elderly men and women. Psychoneuroendocrinology. 2004; 29(3):405-421. [PubMed: 14644069]

Hogervorst E, Matthews FE, Brayne C. Are optimal levels of testosterone associated with better cognitive function in healthy older women and men? Biochim Biophys Acta. 2010; 1800(10): 1145-1152. [PubMed: 20060437]

Holtzer R, Goldin Y, Zimmerman ME. Comparison of robust versus conventional norms of neuropsychological tests in aging. J Int Neuropsychol Soc. 2007; 13(Supplement S1):4.

Hoogerduijn JG, Schuurmans MJ, Duijnstee MS, de Rooij SE, Grypdonck MF. A systematic review of predictors and screening instruments to identify older hospitalized patients at risk for functional decline. J Clin Nurs. 2007; 16(1):46-57. [PubMed: 17181666]

Jak AJ, Bangen KJ, Wierenga CE, Delano-Wood L, Corey-Bloom J, Bondi MW. Contributions of neuropsychology and neuroimaging to understanding clinical subtypes of mild cognitive impairment. Int Rev Neurobiol. 2009; 84:81-103. [PubMed: 19501714]

Janowsky JS. Thinking with your gonads: testosterone and cognition. Trends Cogn Sci. 2006; 10(2): 77-82. [PubMed: 16386941]

Jover-Mengual T, Zukin RS, Etgen AM. MAPK signaling is critical to estradiol protection of CA1 neurons in global ischemia. Endocrinology. 2007; 148(3):1131-1143. [PubMed: 17138646]

Lamberts SW, van den Beld AW, van der Lely AJ. The endocrinology of aging. Science. 1997; 278(5337):419-424. [PubMed: 9334293]

Lipton RB, Katz MJ, Kuslansky G, Sliwinski MJ, Stewart WF, Verghese J, et al. Screening for dementia by telephone using the memory impairment screen. J Am Geriatr Soc. 2003; 51(10): 1382-1390. [PubMed: 14511157]

Lombardi G, Zarrilli S, Colao A, Paesano L, Di Somma C, Rossi F, et al. Estrogens and health in males. Mol Cell Endocrinol. 2001; 178(1-2):51-55. [PubMed: 11403894]

Manly JJ, Merchant CA, Jacobs DM, Small SA, Bell K, Ferin M, et al. Endogenous estrogen levels and Alzheimer's disease among postmenopausal women. Neurology. 2000; 54(4):833-837. [PubMed: 10690972]

Martin DM, Wittert G, Burns NR, Haren MT, Sugarman R. Testosterone and cognitive function in ageing men: data from the Florey Adelaide Male Ageing Study (FAMAS). Maturitas. 2007; 57(2): 182-194. [PubMed: 17287097]

Matousek RH, Sherwin BB. Sex steroid hormones and cognitive functioning in healthy, older men. Horm Behav. 2010; 57(3):352-359. [PubMed: 20079740]

Matsumoto A. Synaptogenic action of sex steroids in developing and adult neuroendocrine brain. Psychoneuroendocrinology. 1991; 16(1-3):25-40. [PubMed: 1961842] 
McEwen BS. Clinical review 108: The molecular and neuroanatomical basis for estrogen effects in the central nervous system. J Clin Endocrinol Metab. 1999; 84(6):1790-1797. [PubMed: 10372665]

Moffat SD, Zonderman AB, Metter EJ, Blackman MR, Harman SM, Resnick SM. Longitudinal assessment of serum free testosterone concentration predicts memory performance and cognitive status in elderly men. J Clin Endocrinol Metab. 2002; 87(11):5001-5007. [PubMed: 12414864]

Muller M, Aleman A, Grobbee DE, de Haan EH, van der Schouw YT. Endogenous sex hormone levels and cognitive function in aging men: is there an optimal level? Neurology. 2005; 64(5):866871. [PubMed: 15753424]

Muller M, van den Beld AW, Grobbee DE, de Jong FH, Lamberts SW. Sex hormones and cognitive decline in elderly men. Psychoneuroendocrinology. 2009; 34(1):27-31. [PubMed: 18845400]

Osterlund MK, Grandien K, Keller E, Hurd YL. The human brain has distinct regional expression patterns of estrogen receptor alpha mRNA isoforms derived from alternative promoters. $\mathbf{J}$ Neurochem. 2000; 75(4):1390-1397. [PubMed: 10987818]

Peiris AN, Stagner JI, Plymate SR, Vogel RL, Heck M, Samols E. Relationship of insulin secretory pulses to sex hormone-binding globulin in normal men. J Clin Endocrinol Metab. 1993; 76(2): 279-282. [PubMed: 8432769]

Petersen RC, Smith GE, Waring SC, Ivnik RJ, Tangalos EG, Kokmen E. Mild cognitive impairment: clinical characterization and outcome.[erratum appears in Arch Neurol 1999 Jun;56(6):760]. Archives of Neurology. 1999; 56(3):303-308. [PubMed: 10190820]

Sarazin M, Berr C, De Rotrou J, Fabrigoule C, Pasquier F, Legrain S, et al. Amnestic syndrome of the medial temporal type identifies prodromal AD: a longitudinal study. Neurology. 2007; 69(19): 1859-1867. [PubMed: 17984454]

Savaskan E, Olivieri G, Meier F, Ravid R, Muller-Spahn F. Hippocampal estrogen beta-receptor immunoreactivity is increased in Alzheimer's disease. Brain Res. 2001; 908(2):113-119. [PubMed: 11454321]

Sheikh JI, Yesavage JA. Geriatric Depression Scale (GDS): recent evidence and development of a shorter version. Clinical Gerontologist. 1986; 5:165-173.

Sodergard R, Backstrom T, Shanbhag V, Carstensen H. Calculation of free and bound fractions of testosterone and estradiol-17 beta to human plasma proteins at body temperature. J Steroid Biochem. 1982; 16(6):801-810. [PubMed: 7202083]

Steinberg BA, Bieliauskas LA, Smith GE, Ivnik RJ. Mayo's Older Americans Normative Studies: Age- and IQ-Adjusted Norms for the Wechsler Memory Scale--Revised. Clin Neuropsychol. 2005; 19(3-4):378-463. [PubMed: 16120536]

Stellato RK, Feldman HA, Hamdy O, Horton ES, McKinlay JB. Testosterone, sex hormone-binding globulin, and the development of type 2 diabetes in middle-aged men: prospective results from the Massachusetts male aging study. Diabetes Care. 2000; 23(4):490-494. [PubMed: 10857940]

Thilers PP, Macdonald SW, Herlitz A. The association between endogenous free testosterone and cognitive performance: a population-based study in 35 to 90 year-old men and women. Psychoneuroendocrinology. 2006; 31(5):565-576. [PubMed: 16487665]

Thurston RC, Sowers MR, Sternfeld B, Gold EB, Bromberger J, Chang Y, et al. Gains in body fat and vasomotor symptom reporting over the menopausal transition: the study of women's health across the nation. Am J Epidemiol. 2009; 170(6):766-774. [PubMed: 19675142]

Tsolaki M, Grammaticos P, Karanasou C, Balaris V, Kapoukranidou D, Kalpidis I, et al. Serum estradiol, progesterone, testosterone, FSH and LH levels in postmenopausal women with Alzheimer's dementia. Hell J Nucl Med. 2005; 8(1):39-42. [PubMed: 15886752]

van den Beld AW, de Jong FH, Grobbee DE, Pols HA, Lamberts SW. Measures of bioavailable serum testosterone and estradiol and their relationships with muscle strength, bone density, and body composition in elderly men. J Clin Endocrinol Metab. 2000; 85(9):3276-3282. [PubMed: 10999822]

Vandenput L, Ohlsson C. Estrogens as regulators of bone health in men. Nat Rev Endocrinol. 2009; 5(8):437-443. [PubMed: 19528961]

Wechsler, D. Wechsler Memory Scale - Revised Manual. San Antonio, TX: The Psychological Corporation; 1987.

Wechsler, D. Wechsler Adult Intelligence Scale. 3. New York: The Psychological Corporation; 1997. 
Wolf OT, Kirschbaum C. Endogenous estradiol and testosterone levels are associated with cognitive performance in older women and men. Horm Behav. 2002; 41(3):259-266. [PubMed: 11971659]

Wu FC, Tajar A, Beynon JM, Pye SR, Silman AJ, Finn JD, et al. Identification of late-onset hypogonadism in middle-aged and elderly men. N Engl J Med. 2010; 363(2):123-135. [PubMed: 20554979]

Xu H, Gouras GK, Greenfield JP, Vincent B, Naslund J, Mazzarelli L, et al. Estrogen reduces neuronal generation of Alzheimer beta-amyloid peptides. Nat Med. 1998; 4(4):447-451. [PubMed: 9546791]

Yaffe K, Barnes D, Lindquist K, Cauley J, Simonsick EM, Penninx B, et al. Endogenous sex hormone levels and risk of cognitive decline in an older biracial cohort. Neurobiol Aging. 2007; 28(2):171178. [PubMed: 17097195]

Yaffe K, Lui LY, Zmuda J, Cauley J. Sex hormones and cognitive function in older men. J Am Geriatr Soc. 2002; 50(4):707-712. [PubMed: 11982672]

Zimmerman ME, Brickman AM, Paul RH, Grieve SM, Tate DF, Gunstad J, et al. The relationship between frontal gray matter volume and cognition varies across the healthy adult lifespan. Am J Geriatr Psychiatry. 2006; 14(10):823-833. [PubMed: 17001022]

Zimmerman ME, Pan JW, Hetherington HP, Katz MJ, Verghese J, Buschke H, et al. Hippocampal neurochemistry, neuromorphometry, and verbal memory in nondemented older adults. Neurology. 2008; 70(18):1594-1600. [PubMed: 18367703] 


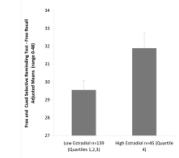

Figure 1.

Bar Graph Showing FCSRT Means (Adjusted for Age, Education, BMI, and Cardiovascular Comordidities) and Standard Errors by Total Estradiol Group in Older Men. 


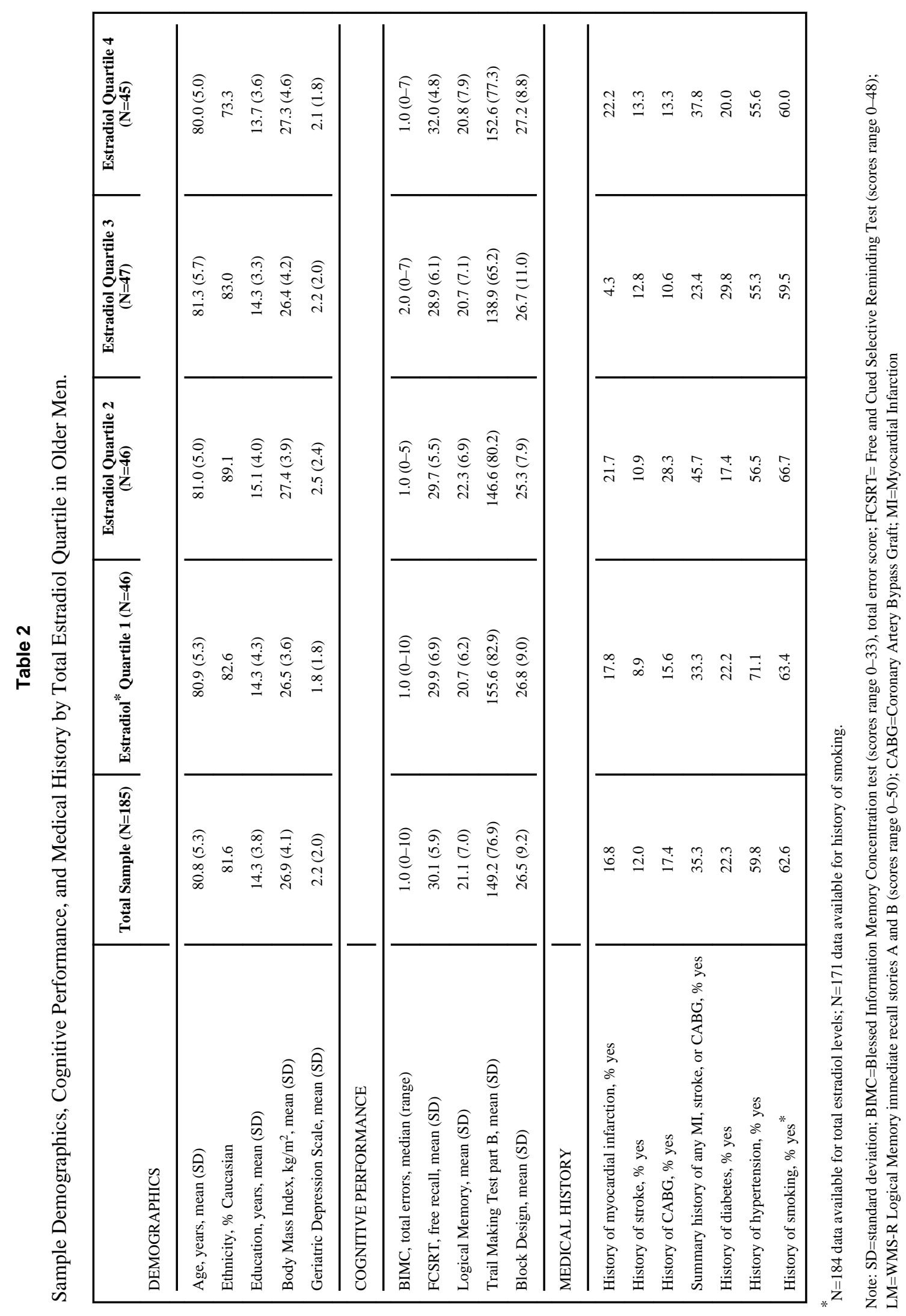




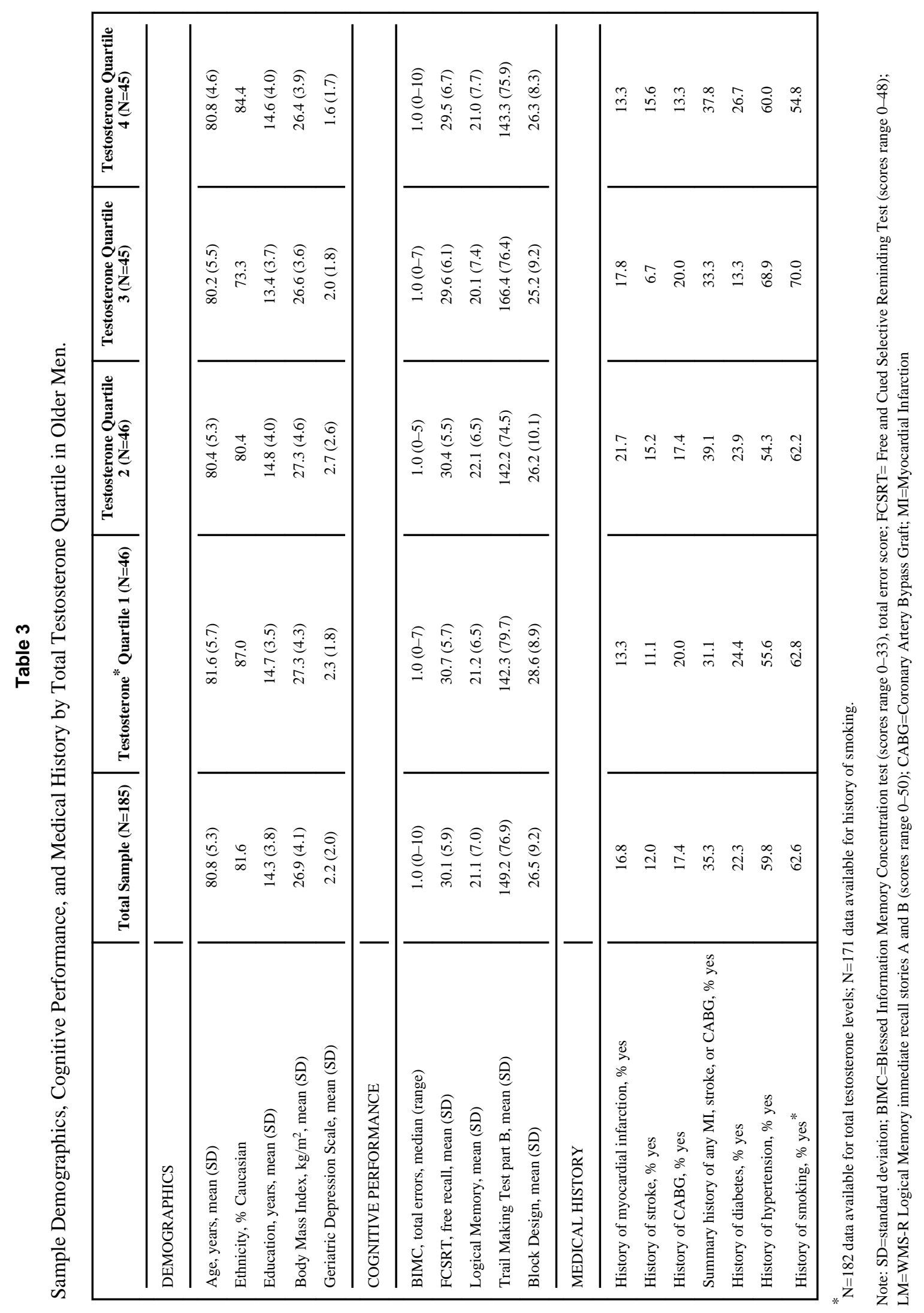

Brain Cogn. Author manuscript; available in PMC 2012 June 1. 


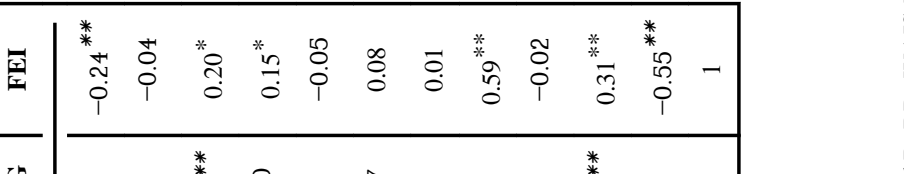

U

$+\sum_{\Sigma}^{\dot{\Phi}}$

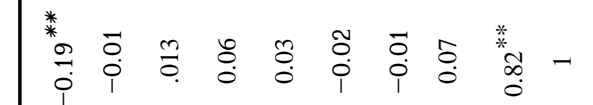

